Lepr Rev (1995) 66, 235-238.

\title{
Hand wounds in leprosy patients
}

\author{
D. SOARES \& N. DESAR \\ Anandaban Leprosy Hospital, P.O. Box 151, Kathmandu, Nepal
}

Accepted for publication 27 March 1995

Summary This study assessed the causes, duration and site of hand wounds seen among patients in order to try and improve the delivery of self-care teaching to patients with anaesthetic hands. Seventy-seven patients with 102 affected hand surfaces were assessed. The commonest cause was a burn from a tea glass. The average duration of the wound was 2 weeks. Most patients had a single current wound and $62 \%$ of wounds were on the palmar surface.

\section{Introduction}

The aim of this study was to identify the common causes of hand wounds among leprosy patients in Nepal with a view to focusing our health education in order to reduce the future damage to insensitive hands. ${ }^{1}$

\section{Methods}

All patients with hand wounds over a six-week period were notified to ND by the other physiotherapy technicians and our other clinical staff. To simplify assessment we assessed each hand as two surfaces (dorsal and palmar). Thus each patient could have a maximum of 4 surfaces affected. Each surface was tested at 14 standard sites (Figure 1). Patients who presented as outpatients as well as inpatients were assessed. All assessments were done by one physiotherapy technician (ND). They had a sensory map (using a 2-g nylon filament) of the affected surface recorded as well as the wounds and any bone loss charted. The patient's name, hospital number, cause of wound and surface was also recorded on our standard sensory testing forms.

\section{Results}

We assessed 77 (58 male, 19 female) consecutive patients who had 102 affected hand surfaces. Of these, 60 patients had 1 surface affected, 11 patients had 2 surfaces affected, 4 patients had 3 surfaces affected and 2 patients had all 4 surfaces affected. 


\section{Right Palmar}

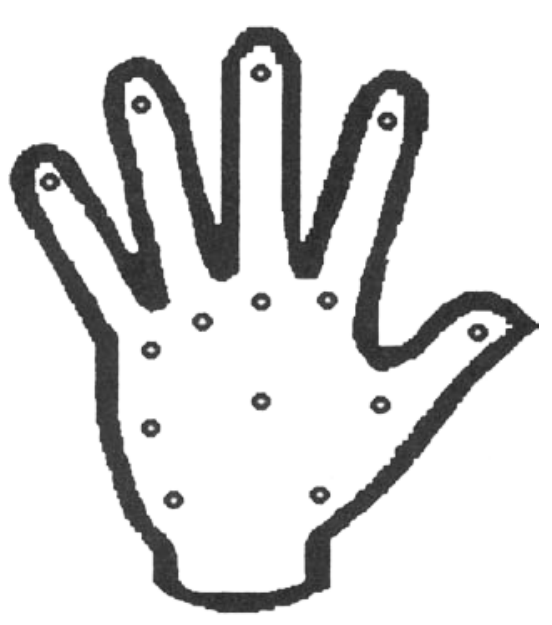

Right Dorsum

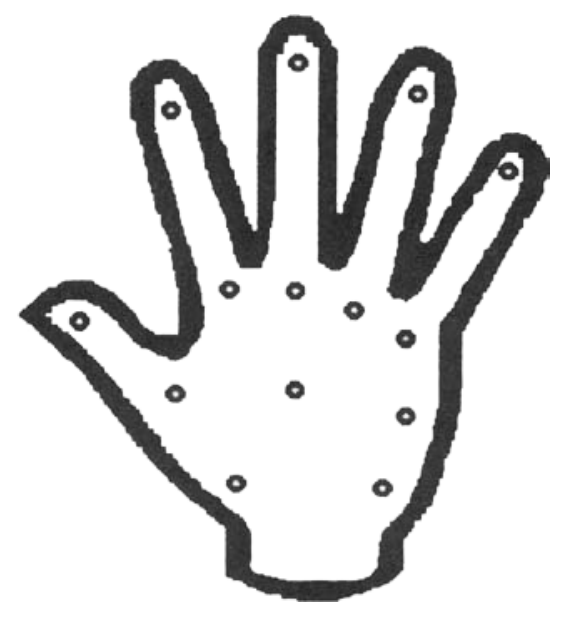

Figure 1. Hand sensory map showing standard testing sites.

All the rest of the data relates to surfaces affected (as c.f. patients).

The left hand was affected in $54 \%$ and the right hand in $46 \%$. The dorsal surface was affected in $32 \%$ and the palmar surface in $68 \%$.

Seventy-one percent of affected surfaces were fully anaesthetic, whereas only $6 \%$ of affected surfaces had anaesthesia at less than 3 sites (out of 14). There were no wounds at sites with normal sensation, i.e. the risk of getting a wound is directly related to sensory loss as judged by a $2-\mathrm{g}$ nylon filament.

Most patients (79\%) had only 1 wound. However $13 \%$ had 3 or more wounds, see Table 1 .

In $70 \%$ of surfaces patients had not lost any bone from the hand. Each testing site where the digit was shortened or bone lost was counted (Figure 2). Eighteen percent had lost bone at $1-3$ sites (out of 14), $13 \%$ had lost bone at 4 or more sites.

In $50 \%$ of patients the duration of the wound was less than 2 weeks, $30 \%$ of patients presented 2-4 weeks after onset of the wound, and $20 \%$ of patients had wounds for more than 4 weeks.

The commonest cause of wounds was a burn (61\%) either from a hot object $(43 \%$ of surfaces), usually a tea glass, or directly from a fire (18\% of surfaces). The other causes are listed in Table 2. In $10 \%$ of patients the cause was not found.

Seventy-two percent of patients had open wounds. The remainder $(28 \%)$ when seen had closed blisters.

Table 1

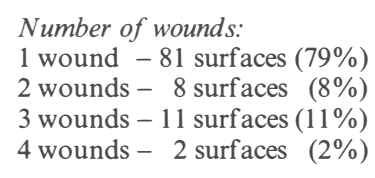




\section{Right Palmar}

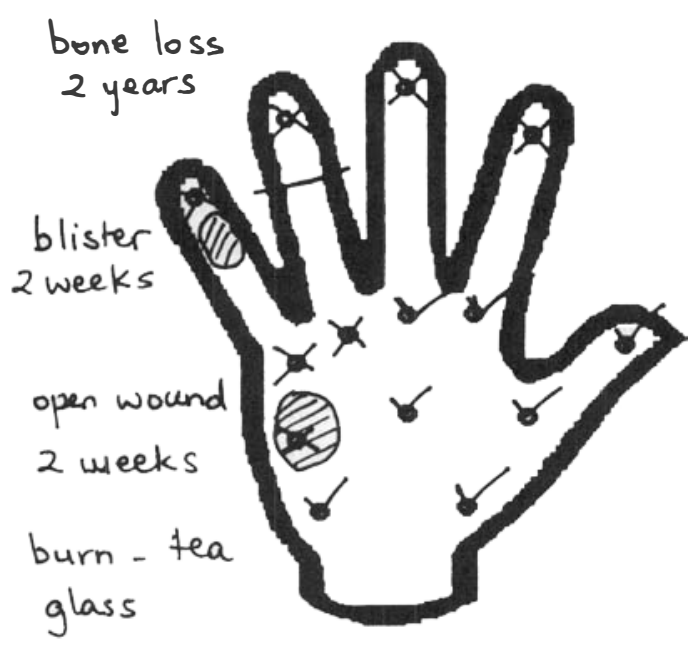

\section{Right Dorsum}

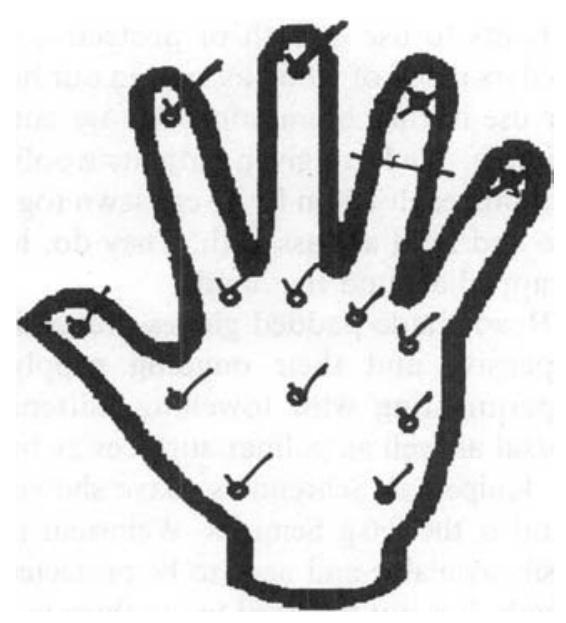

Key: $\checkmark=$ feels, $\quad X=$ does not feel, $\quad$ open crack,
$=$ wound

Figure 2. Sample patient map showing anaesthesia, wounds and bone loss.

There was no significant difference between males and females in any of the following: number of wounds, touch sensation to 2-g nylon filament, bone loss, cause of wound, side affected, surface, number of sites or duration of wound.

There was no significant difference in number of wounds, side affected, surface, number of sites for wounds caused by different categories, e.g., burn versus rough object.

\section{Discussion}

Ascertaining the cause of wounds in patients with insensitive hands is always difficult. It takes patience on the part of staff not to accept the standard 'It just came by itself' and to search with the patient for the true cause. Although time consuming this is the essential first step on the road to prevention of further injuries.

\section{Table 2}

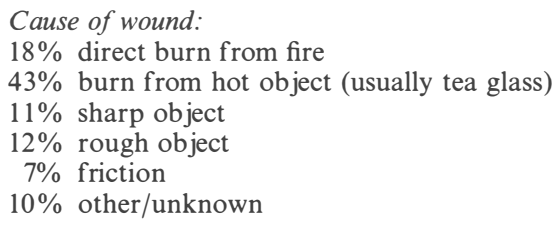


The commonest cause in our study of hand wounds is a burn from a hot object (usually a tea glass). There is great scope for prevention of these injuries by teaching patients to use a cloth or protective glove to hold hot objects. ${ }^{1}$ Traditional tea glass holders made of wood as used in our hospital are fine for inpatients but not as acceptable for use in the community. We are currently trying out what varieties might be locally suitable. We have given patients woollen square gloves (made from two knitted squares of wool, each $15 \mathrm{~cm}$ by $15 \mathrm{~cm}$ sewn together on 3 sides) but these have proved difficult to use and hold a glass with. They do, however, protect the fingertips more than a cloth wrapped around the hand.

Readymade padded gloves are available in the tourist shops in Kathmandu but are expensive and their ongoing supply to our patients is not sustainable. We are experimenting with towelling mittens. When cooking, patients need to protect the dorsal as well as palmar surfaces as both are almost equally vulnerable if insensitive.

Kuipers \& Schreuders ${ }^{2}$ have shown that the threshold for protective sensation in the hand is the $2.0 \mathrm{~g}$ Semmes-Weinstein monofilament (SWM). However filaments are not easily available and need to be protected from being kinked as well as being of a standard length. It is not practical to use them in integrated field programmes where care is provided by basic health service staff. We are testing different simple methods of testing (such as using the weight of a ballpen inclined at an angle) to see if they are as reproducible as the SWM.

We have also re-evaluated our teaching methods and hope to use more role-playing and actual demonstrations by patients rather than the traditional 'health talk' as a method of improving patient understanding and practice of hand self-care.

Most patients $(70 \%)$ in our study have not had any bone loss due to complications of hand wounds. Further health education needs to be concentrated on these patients to ensure that they continue to preserve their hands. They need to be followed up to see which if any develop bone loss over a longer period of time. The $13 \%$ of patients who had lost bone at 4 or more sites are less likely to be able to stay wound free in the future but still deserve the time and effort spent in teaching them self-care.

\section{Conclusion}

This study of causes of hand wounds can help staff to gain an understanding of specific dangers to insensitive hands and help to focus patient training towards prevention of further injury.

\section{Acknowledgments}

Our thanks to Jean Watson of The Leprosy Mission International for her advice, encouragement and enthusiasm.

\section{References}

${ }^{1}$ Watson JM. Preventing Disability in Leprosy Patients pp 67-72. The Leprosy Mission International. 1986.

${ }^{2}$ Kuipers M, Schreuders T. The predictive value of sensation testing in the development of neuropathic ulceration on the hands of leprosy patients. Lepr Rev, 1994; 65: 253-261. 